\title{
Taxonomic analysis of Sorangium strains based on HSP60 and 16S rRNA gene sequences and morphology
}

\author{
De-Ming Jiang, Lin Zhao, Cui-Ying Zhang, Jian Li, Zhi-Jie Xia, Jing Wang, \\ Zhi-Hong Wu and Yue-Zhong Li
}

Correspondence

Yue-Zhong Li

lilab@sdu.edu.cn

\author{
State Key Laboratory of Microbial Technology, College of Life Science, Shandong University, Jinan \\ 250100, PR China
}

\begin{abstract}
The taxonomy of myxobacteria is based mainly on their morphological characteristics. The genus Sorangium belongs to the myxobacterial suborder Sorangiineae. Strains in the genus were classified either as one species, Sorangium cellulosum, by ignoring divergent morphological characteristics, or into several species; however, the latter classification is based on some dubious morphological characteristics and is inconsistent with the phylogeny constructed from 16S rRNA gene sequences. In this study, two HSP60 ( $g r o E L 1$ and groEL2) genes were amplified and sequenced from 22 Sorangium strains. The groEL 1 and groEL2 gene sequences were highly conserved in Sorangium strains, suggesting that these two paralogous genes both play important roles in the life cycle. The phylogeny constructed by the groEL genes was rather consistent with the morphological characteristics of sporangioles. Including information from the phylogenetic analysis and morphological characteristics, it is suggested that the genus Sorangium includes two species.
\end{abstract}

Classical taxonomy of myxobacteria was established based mainly on morphological characteristics (McCurdy, 1989; Reichenbach \& Dworkin, 1992). While stable morphological characteristics such as shapes of vegetative cells and myxospores are consistent with the classification of suborders, the genera or species of myxobacteria have generally been divided on the basis of some dubious morphological characteristics such as the structure and colour of fruiting bodies (Reichenbach, 2005; Shimkets et al., 2006).

The genus Sorangium is located in the suborder Sorangiineae. Its members are remarkable among the 19 myxobacterial genera not only for their unique ability to degrade cellulosic materials (Reichenbach, 2005; Hou et al., 2006) but also for their excellent capacity to produce various bioactive metabolites (Gerth et al., 2003). Sorangium strains have been classified either into one determinate species, Sorangium cellulosum, suggested to contain many variants, or into several species (Reichenbach, 2005), or were tentatively divided into three

The GenBank/EMBL/DDBJ accession numbers for the sequences reported in this paper are EU240489-EU240498 and EU240530EU240533 (16S rRNA genes), EU240499-EU240520 (groEL 1) and EU240521-EU240529 (groEL2).

Micrographs of Sorangium strains and an alignment of $16 \mathrm{~S}$ rRNA gene sequences are available as supplementary material with the online version of this paper. species based mainly on the colour of fruiting bodies, whereby strains with orange, dark brown and nearly black fruiting bodies were named 'Sorangium compositum', S. cellulosum and 'Sorangium nigrum', respectively (Shimkets et al., 2006). However, the names 'S. compositum' and 'S. nigrum' have not been validly published (http://www. bacterio.cict.fr/s/sorangium.html). Although the present morphology-based taxonomy of the genus Sorangium is coherent, species are often inconsistent with the phylogenetic results from 16S rRNA gene sequences. The $60 \mathrm{kDa}$ heat-shock protein (HSP60; also known as GroEL) belongs to the chaperonin family (Lin \& Rye, 2006). HSP60 is ubiquitous and highly conserved in bacteria, and thus is being used more frequently to infer eubacterial phylogenies (Karlin \& Brocchieri, 2000). The groEL gene is present as a single copy in most bacterial species, but two or more copies are found in some bacteria (Goyal et al., 2006). In this paper, two different HSP60 genes (groEL1 and groEL2) were amplified from Sorangium strains. Phylogenetic analyses based on HSP60 and 16S rRNA gene sequences were compared comprehensively with the morphological taxonomy for 22 Sorangium strains.

\section{Morphologies of the Sorangium strains}

The morphological properties of some of the 22 Sorangium strains used in this study are listed in Table 1 . The strains were routinely cultivated on CNST medium, containing (w/v) $0.05 \% \quad \mathrm{KNO}_{3}, \quad 0.025 \% \mathrm{Na}_{2} \mathrm{HPO}_{4} .12 \mathrm{H}_{2} \mathrm{O}, 0.1 \%$ 


\section{Table 1. Major morphological characteristics of Sorangium strains}

Morphological characteristics of the nine other strains included in this study, So9987-5, So9735-22, So9881, So9721-1, So9711-1, So9733-1, So0089-1, So9741 and So ce26, were reported previously (Yan et al., 2003). Strains DSM $14627^{\mathrm{T}}$ and DSM 14731 were obtained from the Deutsche Sammlung von Mikroorganismen und Zellkulturen. Strain So ce26 was provided by Hans Reichenbach. The other strains shown, isolated and maintained by the authors, were chosen from hundreds to represent strains with diverse morphological characteristics. NA, Not applicable.

\begin{tabular}{|c|c|c|c|c|c|c|c|}
\hline \multirow[t]{2}{*}{ Strain } & \multicolumn{2}{|c|}{ Size $(\mu \mathrm{m})$} & \multicolumn{2}{|c|}{ Sporangiole } & \multicolumn{3}{|c|}{ Fruiting body } \\
\hline & Vegetative cells & Myxospores & Shape & Size $(\mu \mathrm{m})$ & Colour & Texture & Distribution \\
\hline So0087-23 & $4-8 \times 0.8-1$ & $2-3 \times 0.8-1$ & Spherical, in parcels & $10-15$ & Red-orange & Rigid & Whole \\
\hline So0085-1 & $4-7 \times 0.8-1$ & $2-4 \times 0.8-1$ & Spherical, in parcels & $10-15$ & Red-orange & Rigid & Rusted region \\
\hline So0157-1 & $6-8 \times 0.8-1$ & $2-3 \times 0.8-1$ & Spherical, in parcels & $10-15$ & Red-orange & Soft & Whole \\
\hline So0133-4 & $5-6 \times 0.8-1$ & $1-2 \times 0.8-1$ & Spherical, in parcels & $10-15$ & Yellow & Soft & Whole \\
\hline So0081-1 & $4-6 \times 0.8-1$ & $2-3 \times 0.8-1$ & Spherical, in parcels & $15-20$ & Yellow & Rigid & Whole \\
\hline So9734-1 & $4-7 \times 0.8-1$ & $2-3 \times 0.8-1$ & Spherical, in parcels & $10-15$ & Red-orange & Soft & Whole \\
\hline So0139-5 & $4-6 \times 0.8-1$ & $2-3 \times 0.8-1$ & Spherical, unpacked & $15-25$ & NA & NA & NA \\
\hline So0184-1 & $4-7 \times 0.8-1$ & $2-3 \times 0.8-1$ & Spherical, in parcels & $10-20$ & Yellow & Soft & Whole \\
\hline
\end{tabular}

$\mathrm{MgSO}_{4} \cdot 7 \mathrm{H}_{2} \mathrm{O}, 0.001 \% \mathrm{FeCl}_{3} \cdot 6 \mathrm{H}_{2} \mathrm{O}, 1.5 \%$ agar and $1 \mathrm{ml}$ trace elements solution $\mathrm{l}^{-1}$ (Yan et al., 2003). Morphologies were determined using the methods described in a previous report (Yan et al., 2003).

The sporangioles, which contain many embedded myxospores, were either spherical or polyhedral in shape and varied in size. They were often packed into macroscopic fruiting bodies, but unpacked sporangioles were also found in some strains, such as So0157-52 and So9721-1. Most of the 22 strains had yellow to red-orange fruiting bodies, while some strains, i.e. So0089-1, So ce26, So9741 and DSM 14731, had a deeper colour of brown or black. Fruiting mostly appeared with the whole swarm on partially or completely degraded filter paper, while some strains formed fruiting bodies only in the swarm centre (So9741), at the swarm edge (So9881) or at undegraded places far beyond the degraded regions (So ce26). Although the characteristics of the fruiting bodies have been one of the important taxonomic criteria for the classification of species in Sorangium, this characteristic was not stable and either degenerated or was lost in many strains after rounds of cultivation. Some strains, such as So9987-5, So0007-16 and So0139-5, had no sporangiole structures when they were initially isolated. Members of the Sorangiineae have been shown to be able to etch agar (Reichenbach \& Dworkin, 1992), but the ability varied greatly among different strains. On filter paper-CNST medium, the extension of sorangial swarms also varied among the strains. All of the 22 strains had the ability to degrade cellulose, and a piece of filter paper with an area of $1 \mathrm{~cm}^{2}$ was degraded completely after 7-14 days of cultivation at $30{ }^{\circ} \mathrm{C}$. Supplementary Fig. S1, available in IJSEM Online, demonstrates the typical morphologies, especially the sporangioles, of the Sorangium strains.

\section{Phylogenetic analysis based on 16S rRNA gene sequences}

Extraction of genomic DNA from cells and PCR amplification and sequencing of $16 \mathrm{~S}$ rRNA genes were performed using previously described methods ( $\mathrm{Li}$ et al., 2002). For phylogenetic analysis, nucleotide sequences were aligned using the CLUSTAL_X computer program (version 1.83; Thompson et al., 1997). The aligned sequences were refined and then analysed phylogenetically by distance/ neighbour-joining programs with the Poisson correction distance model in the MEGA3.1 software package (Kumar et al., 2004) to infer their phylogenetic relationships. The phylogenetic relationships of the sequences were also confirmed by the likelihood and parsimony programs in the PHYLIP software package (v. 3.61) (Felsenstein, 2001). Bootstrapping support for the interior branch lengths of the tree was obtained from 1000 replicates. Reference sequences were extracted from GenBank.

Fig. 1(a) shows the phylogenetic tree constructed from the $16 \mathrm{~S}$ rRNA gene sequences of all the Sorangium strains examined. The phylogenetic tree was highly consistent with that constructed with 10 Sorangium strains by Yan et al. (2003). The sequence similarity of the Sorangium strains to the cellulolytic species Byssovorax cruenta was 94-96\%. Within Sorangium, the divergence was less than $2.3 \%$, except strain So9741, which exhibited the lowest sequence interstrain similarity, of $96.8 \%$, in comparison with So ce26. The topology of the tree separated the strains into 
(a)

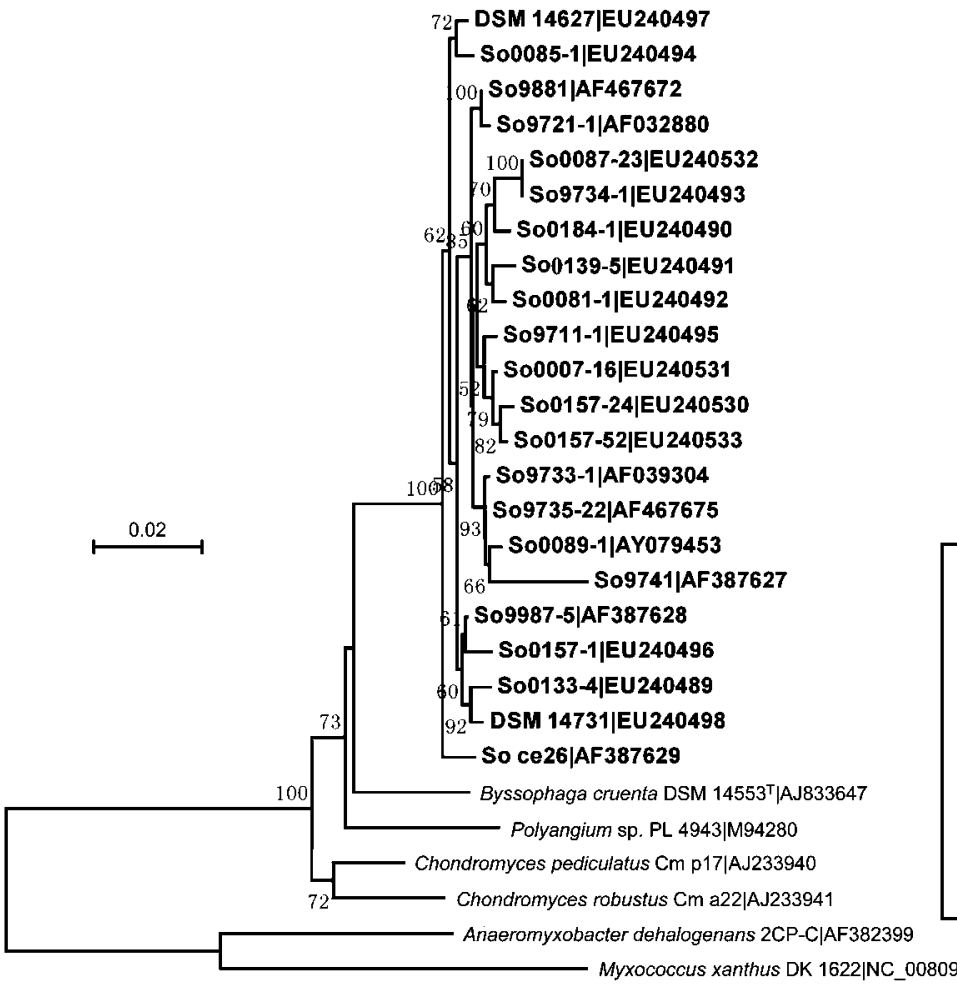

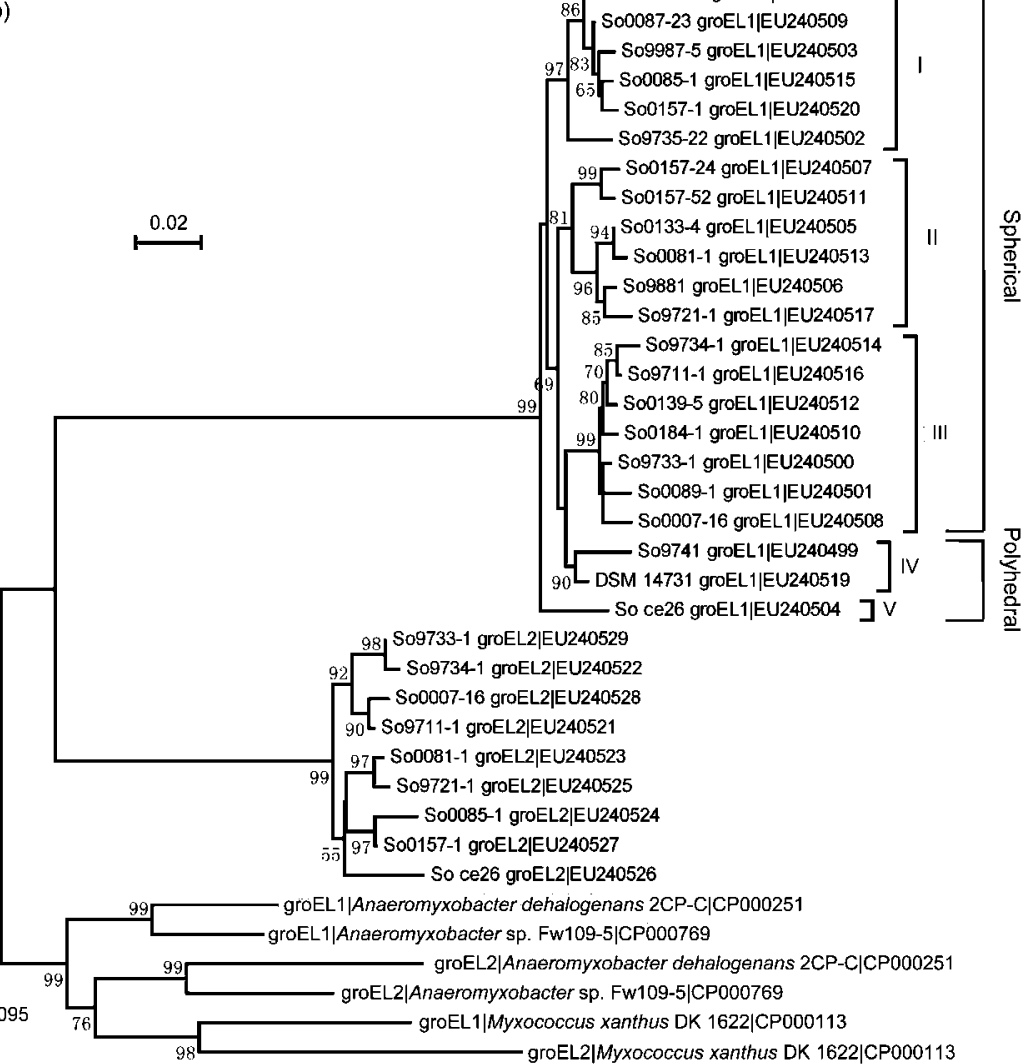

Fig. 1. Phylogenetic analysis based on $16 \mathrm{~S}$ rRNA gene sequences (a) and HSP60 gene sequences (b) of 22 Sorangium strains and related reference myxobacteria strains. Aligned sequences of the two types of HSP60 gene (groEL1 and groEL2) were extracted to the same length of about 600 bp. In (b), branches mentioned in the text are indicated by the roman numerals $\mathrm{I}-\mathrm{V}$, and groups of spherical and polyhedral sporangiole-forming strains are indicated. Bars, 2 changes per 100 nucleotide positions. Numbers on branch nodes indicate bootstrap support percentages based on 1000 replicates. 
four groups. However, this separation was not supported by morphological characteristics.

To analyse the nucleotide changes in the 16S rRNA gene sequences of Sorangium strains in detail, the sequences were aligned corresponding to the conserved and variable regions of bacterial 16S rRNA gene sequences, and divergent nucleotides were highlighted (Supplementary Fig. S2). There were 96 sites in the sequence that contained one or two divergent nucleotides, including many unique inserts. The divergent sites were scattered along the $16 \mathrm{~S}$ rRNA gene sequence, not only in the variable regions but also in conserved regions. Compared with the other 21 strains, So9741 had many unique bases in conserved regions C3, C4, C5 and C6. This clearly indicated that strain So9741 should be separated from the other strains as a member of a different species. Although So ce26 formed a separate clade from the others in the phylogenetic tree, the strain did not contain many unique bases. The divergent sites of So ce26 appeared mainly in the variable regions V2 and V4. It is also interesting to see that, in So9987-5, there were nine unique two-base inserts in the $16 \mathrm{~S}$ rRNA gene sequence, but the phylogenetic tree did not reflect this change. Obviously, gaps had been removed before phylogenetic analysis and were not included. The results revealed that the phylogeny constructed from the Sorangium 16S rRNA gene sequences provides little information for the classification of species.

\section{Phylogenetic analysis based on HSP60 sequences}

Screening the published myxobacterial genomes revealed two copies of the HSP60 gene (groEL1 and groEL2). The degenerate primers $\mathrm{H} 279$ and $\mathrm{H} 280$ described by Goh et al. (1996) were first used for the amplification of the conserved segment of groEL1 (with an expected length of about $600 \mathrm{bp}$ ) from the Sorangium strains. The $25 \mu \mathrm{l}$ PCR mixture consisted of approximately $300 \mathrm{ng}$ DNA template, $2.5 \mathrm{nmol}$ each dNTP, $2.5 \mathrm{U}$ Taq DNA polymerase, $1 \times$ GC buffer and 50 pmol each of primers $\mathrm{H} 279$ and $\mathrm{H} 280$. The thermal cycling conditions were as follows: $96{ }^{\circ} \mathrm{C}$ for $3 \mathrm{~min}$ followed by 30 cycles of $94{ }^{\circ} \mathrm{C}$ for $1 \mathrm{~min}, 46{ }^{\circ} \mathrm{C}$ for $1 \mathrm{~min}$ and $72{ }^{\circ} \mathrm{C}$ for $1 \mathrm{~min}$. Finally, the reaction was maintained at $72{ }^{\circ} \mathrm{C}$ for $10 \mathrm{~min}$. This degenerate primer set produced positive results from 14 of the 22 Sorangium strains. A new Sorangium-specific primer set, HSP60MyxoF and HSP60MyxoR, was designed based on the sequences of the groEL1 genes that were amplified from Sorangium strains by using the $\mathrm{H} 279 / \mathrm{H} 280$ primer set. Our new myxobacteria-specific primers were able to amplify the gene segment from all of the Sorangium strains that we studied using the above PCR conditions with the slight modification of setting the annealing temperature to $70{ }^{\circ} \mathrm{C}$. Two new degenerate primers, HSP60Myхо2F and HSP60Myxo2R, were also designed on the basis of the sequences of putative groEL2 proteins from the three published myxobacteria genomes, those of Myxococcus xanthus DK1622 (GenBank accession no. CP000113), Anaeromyxobacter dehalogenans 2CP-C (CP000251) and Anaeromyxobacter sp. Fw109-5 (CP000769). The PCR amplification was modified by adding 100 pmol of each of the primers to the reaction mixture and setting the annealing temperature to $57{ }^{\circ} \mathrm{C}$ and the extension time to $2.5 \mathrm{~min}$. The primers produced positive results from nine of the 22 Sorangium strains (expected products of about $1440 \mathrm{bp}$ ). The products were analysed by electrophoresis in $1 \%(\mathrm{w} / \mathrm{v})$ agarose gel in $1 \times \mathrm{TAE}$ buffer (containing $50 \mathrm{mM}$ Tris/acetate, $1 \mathrm{mM}$ EDTA, pH 8.0), stained with ethidium bromide and visualized under UV light. After purification using an agarose gel DNA extraction kit (Roche), the amplified DNA sequences were ligated into the pGEM-T Easy vector (Promega) following the protocols recommended by the manufacturer. The ligation mixtures were transferred by electroporation into Escherichia coli JM109 cells using the standard method (Sambrook \& Russell, 2001). Sequencing was performed at the Sangon Sequencing Center (Shanghai, China). The primers employed for amplification of the groEL1 and groEL2 gene sequences are listed in Table 2.

The sequence similarity between the 22 Sorangium groEL1 genes was $94.2-99.6 \%$. The nine groEL2 sequences were also highly similar, with more than $96 \%$ identity. Phylogenetic trees reconstructed from the complete and partial sequences of the groEL2 gene were nearly the same (data not shown), and thus the same segments of groEL1 and groEL2 were combined to reconstruct a phylogenetic tree (Fig. 1b). The two groEL genes were grouped separately. The sequences within each groEL gene showed

Table 2. Primers for PCR amplification of groEL genes from Sorangium strains

Inosine (I) was used to reduce the degeneracy of the primers. Primers were synthesized at Sangon Sequencing Center (Shanghai, China).

\begin{tabular}{|c|c|c|c|}
\hline Primer & Sequence $\left(5^{\prime}-3^{\prime}\right)$ & Target & Reference \\
\hline $\mathrm{H} 280$ & CGCGGGATCC(TC)(TG)I(TC)(TG)ITCICC(AG)AAICCIGGIGC(TC)TT & groEL1 & Goh et al. (1996) \\
\hline HSP60MyxoR & TCGCC(AG)AAGCCGGGGGC(CT)TT(GC)AC & groEL1 & This study \\
\hline HSP60Mухо2F & GT(GC)AC(GC)CT(GC)GG(GC)CC(GC)AAGGG & groEL2 & This study \\
\hline HSP60Myxo2R & CT(GC)GC(GC)GCGTTCTG(GC)AG(GC)GC & groEL2 & This study \\
\hline
\end{tabular}


high levels of similarity, which probably indicates that both genes are important, but play different roles in Sorangium cells. The groEL1 genes were divided into five branches, and phylogenetic analysis based on the nine groEL2 gene sequences was consistent with the tree of groEL1 gene sequences, with only small exceptions within the branches. The similarity between groEL1 and groEL2 in the same Sorangium strain was highly stable, ranging between 71.5 and $73.3 \%$. The sequence similarity between the Sorangium groEL1 sequences and the reference myxobacterial groEL1 sequences varied from 70.6-74.4\% (Anaeromyxobacter strains) to $67.8-70.6 \%$ (M. xanthus DK1622), while the similarity between the Sorangium groEL2 sequences and the reference myxobacterial groEL2 sequences was $73.6-77.7 \%$ (Anaeromyxobacter strains) to $70.1-73.1 \%$ (M. xanthus DK1622). In contrast, the sequence similarity of the two groEL genes was 86.2 and $82.5 \%$ in the two Anaeromyxobacter strains and $83.8 \%$ in M. xanthus DK1622. These results suggest that groEL1 and groEL2 are paralogous genes in myxobacteria. To date, there have been no reports on the functions of the two groEL genes in myxobacteria, although they have been reported in two-dimensional proteome screens (Dahl et al., 2007; Otani et al., 2001). It will be very interesting to discover their functions in the life cycle.

In the groEL1 tree, strain So ce26 was also separated from others, and strain So9741 had the lowest sequence similarity to other strains (94.2-95\%) with the exception of its fellow groEL1 group member DSM 14731 (97.9\%). However, the two trees reconstructed from the groEL and $16 \mathrm{~S}$ rRNA genes were not highly congruent. Comparison of the groEL1 phylogeny and morphological characteristics revealed some significant results. The groEL1 branches were consistent with the shapes of sporangioles, but not with any other morphological characteristics. For example, the sporangioles of the members of branch I were spherical and packed into macroscopic fruiting bodies, while their groEL1 sequences showed similarity of more than $97.2 \%$. Branch IV was composed of two strains, DSM 14731 and So9741, whose sporangioles were polyhedral and were packed to form fruiting bodies. groEL1 sequences divided the Sorangium strains with spherical sporangioles into three subgroups (branches I, II and III) and the strains with polyhedral sporangioles into two subgroups (branches IV and $\mathrm{V}$ ). The results were consistent with the conclusion of our previous paper (Yan et al., 2003), i.e. rather than the colour of the fruiting bodies, sporangiole shape is more important as a morphological taxonomic criterion to separate species in the genus Sorangium. The colour of the fruiting bodies was also consistent with the groEL1-based classification, but there were some exceptions. For instance, strain So0089-1 had black and spherical sporangioles, but was located on branch III.

Sorangium strains have gigantic genomes, more than $13 \mathrm{Mb}$ in S. cellulosum strain So ce56 (Schneiker et al., 2007). Bioinformatic analysis of the genome reveals that a large percentage of the genome is devoted to regulation, which probably supports its complex social lifestyle and thus diversified morphologies. It has long been suggested that there should be more than one species within the genus, and there have been attempts to divide Sorangium strains based on their morphological characteristics (Reichenbach \& Dworkin, 1992; Reichenbach, 2005; Shimkets et al., 2006). However, phylogenetic analysis of $16 \mathrm{~S}$ rRNA gene sequences did not separate the Sorangium strains clearly (Yan et al., 2003). More significantly, the morphological taxonomy of Sorangium is often incompatible with the phylogeny, due to the high similarity of the 16S rRNA gene sequences of Sorangium strains. Interestingly, the two HSP60 genes produced highly similar phylogenetic results, and can be divided into several obvious groups. In the classical taxonomy of the Myxococcales, morphology is the primary basis for classification. The types of fruiting bodies and sporangioles form the basis of classification of genera, and sizes of myxospores or other fine morphological differences have been used to define each species. The phylogenetic tree constructed from the groEL genes is consistent with the morphological characteristics of sporangioles. As mentioned previously, spherical sporangioles have often been observed to coexist with polyhedral sporangioles, which were the variants of the spherical sporangioles, clearly formed by dense packing (Reichenbach, 2005). On the other hand, many Sorangium strains contain only spherical sporangioles, whether they were densely or loosely packed. In the case of strains So ce26, So9741 and DSM 14731, the polyhedral sporangiole shape is a rather stable characteristic even when the strains were cultivated on different media. Additionally, polyhedral sporangioles were usually found mixed with many stuffing substrates, forming fruiting bodies (Yan et al., 2003). The stuffing materials were not able to be observed in fruiting bodies containing spherical or mixed-shaped sporangioles. Thus, the Sorangium strains could be divided, at least, into two groups, containing spherical or polyhedral shapes of sporangioles. For final classification of species or subspecies in Sorangium, more phenotypic as well as genotypic analyses are needed.

\section{Acknowledgements}

This work was supported financially by grants 30671192, 30470001 and 30670028 awarded by the National Natural Science Foundation of China and 2006AA02Z171 of the 863 Programs of China. The authors thank Dr Roberta Greenwood for her help in editing this manuscript. We thank Dr Hans Reichenbach (Helmholtz Centre for Infection Research) for providing strain So ce26.

\section{References}

Dahl, J. L., Tengra, F. K., Dutton, D., Yan, J., Andacht, T. M., Coyne, L., Windell, V. \& Garza, A. G. (2007). Identification of major sporulation proteins of Myxococcus xanthus using a proteomic approach. J Bacteriol 189, 3187-3197.

Felsenstein, J. (2001). PHYLIP (phylogeny inference package) version 3.61. Distributed by the author. Department of Genome Sciences, University of Washington, Seattle, USA. 
Gerth, K., Pradella, S., Perlova, O., Beyer, S. \& Muller, R. (2003). Myxobacteria: proficient producers of novel natural products with various biological activities - past and future biotechnological aspects with the focus on the genus Sorangium. J Biotechnol 106, 233-253.

Goh, S. H., Potter, S., Wood, J. O., Hemmingsen, S. M., Reynolds, R. P. \& Chow, A. W. (1996). HSP60 gene sequences as universal targets for microbial species identification: studies with coagulase-negative staphylococci. J Clin Microbiol 34, 818-823.

Goyal, K., Qamra, R. \& Mande, S. C. (2006). Multiple gene duplication and rapid evolution in the groEL gene: functional implications. J Mol Evol 63, 781-787.

Hou, P. B., Li, Y. Z., Wu, B. H., Yan, Z. C., Yan, B. X. \& Gao, P. J. (2006). Cellulolytic complex exists in cellulolytic myxobacterium Sorangium. Enzyme Microb Technol 38, 273-278.

Karlin, S. \& Brocchieri, L. (2000). Heat shock protein 60 sequence comparisons: duplications, lateral transfer, and mitochondrial evolution. Proc Natl Acad Sci U S A 97, 11348-11353.

Kumar, S., Tamura, K. \& Nei, M. (2004). MEGA3: integrated software for molecular evolutionary genetics analysis and sequence alignment. Brief Bioinform 5, 150-163.

Li, Y. Z., Hu, W., Zhang, Y. Q., Qiu, Z., Zhang, Y. \& Wu, B. H. (2002). A simple method to isolate salt-tolerant myxobacteria from marine samples. J Microbiol Methods 50, 205-209.

Lin, Z. \& Rye, H. S. (2006). GroEL-mediated protein folding: making the impossible, possible. Crit Rev Biochem Mol Biol 41, 211-239.

McCurdy, H. D. (1989). Order Myxococcales Tchan, Pochon and Prévot 1948, 398 ${ }^{\mathrm{AL}}$. In Bergey's Manual of Systematic Bacteriology, vol. 3, pp. 2139-2144. Edited by J. T. Staley, M. P. Bryant, N. Pfennig \& J. G. Holt. Baltimore: Williams \& Wilkins.
Otani, M., Tabata, J., Ueki, T., Sano, K. \& Inouye, S. (2001). Heatshock-induced proteins from Myxococcus xanthus. J Bacteriol 183, 6282-6287.

Reichenbach, H. (2005). Order VIII. Myxococcales Tchan, Pochon and Prévot 1948, $398^{\mathrm{AL}}$. In Bergey's Manual of Systematic Bacteriology, 2nd edn, vol. 2, part C, pp. 1059-1072. Edited by D. J. Brenner, N. R. Krieg, J. T. Staley \& G. M. Garrity. New York: Springer.

Reichenbach, H. \& Dworkin, M. (1992). The myxobacteria. In The Prokaryotes, 2nd edn, pp. 3416-3487. Edited by A. Balows, H. G. Trüper, M. Dworkin, W. Harder \& K. H. Schleifer. New York: Springer.

Sambrook, J. \& Russell, D. W. (2001). Molecular Cloning: a Laboratory Manual, 3rd edn. Cold Spring Harbor, NY: Cold Spring Harbor Laboratory.

Schneiker, S., Perlova, O., Kaiser, O., Gerth, K., Alici, A., Altmeyer, M. O., Bartels, D., Bekel, T., Beyer, S. \& other authors (2007). Complete genome sequence of the myxobacterium Sorangium cellulosum. Nat Biotechnol 25, 1281-1289.

Shimkets, L. J., Dworkin, M. \& Reichenbach, H. (2006). The myxobacteria. In The Prokaryotes: a Handbook on the Biology of Bacteria, 3rd edn, vol. 7, pp. 31-115. Edited by M. Dworkin, S. Falkow, E. Rosenberg, K. H. Schleifer \& E. Stackebrandt. New York: Springer.

Thompson, J. D., Gibson, T. J., Plewniak, F., Jeanmougin, F. \& Higgins, D. G. (1997). The CLUSTAL_X windows interface: flexible strategies for multiple sequence alignment aided by quality analysis tools. Nucleic Acids Res 25, 4876-4882.

Yan, Z. C., Wang, B., Li, Y. Z., Gong, X., Zhang, H. Q. \& Gao, P. J. (2003). Morphologies and phylogenetic classification of cellulolytic myxobacteria. Syst Appl Microbiol 26, 104-109. 